\title{
Semantic Image Retrieval via Active Grounding of Visual Situations
}

\author{
Max H. Quinn ${ }^{1}$, Erik Conser ${ }^{1}$, Jordan M. Witte ${ }^{1}$, and Melanie Mitchell ${ }^{1,2}$ \\ ${ }^{1}$ Portland State University ${ }^{2}$ Santa Fe Institute \\ Email: quinn.max@gmail.com
}

\begin{abstract}
We describe a novel architecture for semantic image retrieval-in particular, retrieval of instances of visual situations. Visual situations are concepts such as "a boxing match," "walking the dog," "a crowd waiting for a bus," or "a game of pingpong," whose instantiations in images are linked more by their common spatial and semantic structure than by low-level visual similarity. Given a query situation description, our architecturecalled Situate-learns models capturing the visual features of expected objects as well the expected spatial configuration of relationships among objects. Given a new image, Situate uses these models in an attempt to ground (i.e., to create a bounding box locating) each expected component of the situation in the image via an active search procedure. Situate uses the resulting grounding to compute a score indicating the degree to which the new image is judged to contain an instance of the situation. Such scores can be used to rank images in a collection as part of a retrieval system. In the preliminary study described here, we demonstrate the promise of this system by comparing Situate's performance with that of two baseline methods, as well as with a related semantic image-retrieval system based on "scene graphs."
\end{abstract}

\section{INTRODUCTION}

The ability to automatically retrieve images with specified semantic properties is a key topic for computer vision. In a world deluged with image data, automated image retrieval has become as important as text search, and progress in this area will have profound impacts in areas as diverse as medical diagnosis, public health, national security, privacy, and personal data organization.

Using deep neural networks, automatic detection of individual objects in images has become remarkably successful [1]. However, in many domains, users need to search for images with more abstract properties, in which multiple objects with specified attributes are related in specific ways. Here are some examples: "a boxing match," "a person walking a dog," "a crowd waiting for a bus," or "a game of ping-pong." Instances of such abstract visual concepts-which we call visual situations - are linked more by their common spatial and semantic structure than by low-level visual similarity or by the specific objects they contain. In general, automatically recognizing instances of a given visual situation is a difficult problem, due to substantial variability in visual features and spatial layout among different instances. Moreover, while state-of-the-art object detection methods often rely on evaluating large numbers of "object proposals" at every location and scale of the image, the combinatorics of such exhaustive evaluations become much worse when the multiple objects, attributes, and possible relationships of a situation need to be considered. And while successful object detection has relied on huge amounts of labeled training data [2], there are few large labeled training sets for visual situations.

In this paper, we describe a novel architecture for retrieving instances of a query visual situation in a collection of images. Our architecture-called Situate-combines objectlocalization models based on visual features with probabilistic models that represent learned multi-object relationships. Situate learns these models from labeled training images. It applies these models to a new image via an active search process that attempts to ground components of the query situation in the image - that is, to create bounding boxes that localize relevant objects and relationships, and that ultimately provide a situation match score for the situation with respect to the image. The match scores can be used to rank the images in the collection with respect to the query: the highest ranking images can be returned to the user. Figure 1 illustrates Situate's training and testing pipeline.

We hypothesize that Situate's learned object and relationship models, used in tandem with its active situation-grounding method, will result in superior image retrieval performance than methods without these components. In this preliminary study, we test this hypothesis by comparing Situate's performance on a challenging visual-situation dataset with that of two baseline methods: a "lesioned" version of Situate that lacks its relationship models and the feedback they provide the system, and an adapted version of the widely used FasterRCNN object-detection method [1]. We also compare Situate's performance with that of a recently proposed semantic image retrieval system [3].

\section{Situate's ARChitecture}

Situate's architecture is inspired by active approaches to perception, in which the perceiver acquires information dynamically, and in which the information acquired continually feeds back to control the perceptual process. In particular, for humans, recognizing a visual situation is an active process that unfolds over time, in which prior knowledge interacts with visual information as it is perceived, in order to guide subsequent eye movements and focus of attention. This interaction of topdown expectations and bottom-up perception enables a human viewer to very quickly locate relevant aspects of the situation [4], [5].

Additionally inspired by the Copycat architecture of Hofstadter and Mitchell [6], Situate attempts to actively ground 

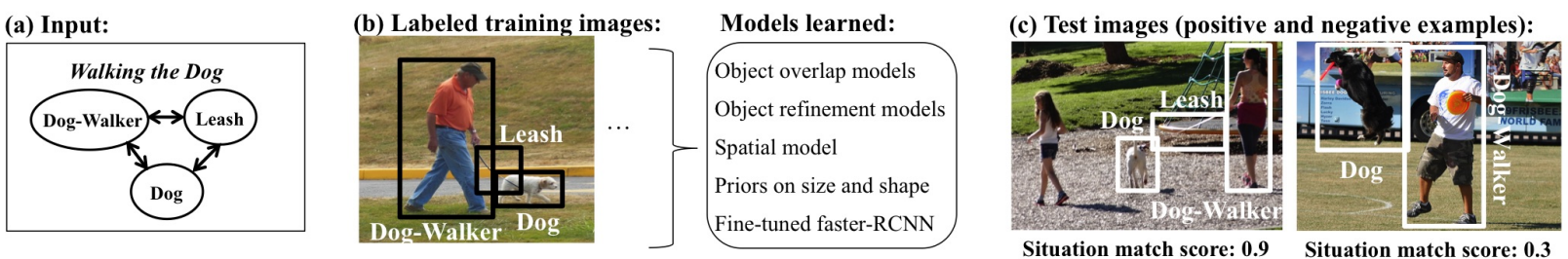

Fig. 1. (Best viewed in color.) Overview of Situate's training and testing pipeline, with the example situation "walking the dog." (a) The user specifies the object categories relevant to the given situation. This simple example includes three expected objects: Dog-Walker, Leash, and Dog; the double-arrows indicate unnamed (i.e., to be learned) relationships among these objects. (b) Situate is also given a set of training images, which include only positive examples of the situation, with relevant objects indicated via human-labeled (black) bounding boxes. From these training examples, Situate learns several types of models. (c) Situate uses its learned models to score test images as instances of the given situation, by attempting to ground the expected object categories and their expected spatial configuration in the image (white boxes).

components of a query situation via the actions of agents in a Workspace. The agents are selected and run over a series of time steps, and create candidate detections by combining bottom-up visual information with top-down expectations. The advantage of this active, temporal approach is that detections made in previous time steps can influence the focus and actions of agents at subsequent time steps.

\section{A. Training}

In attempting to ground situation components in an image, Situate's agents dynamically combine different types of information by employing a set of models that capture expected visual and spatial features of the query situtation. These models are learned from human-labeled training data, illustrated in Figure 1 b). The learned models are the following:

Object-Localization Models: For a given situation query, Situate learns object-localization models for each relevant object category. The input to each object-localization model is an object proposal, which specifies an object category $C$ along with a set of coordinates specifying a bounding box $b$ in an image. For an object-localization model corresponding to category $C$, the model's output is a prediction of the amount $b$ overlaps a ground-truth object of category $C$. Following standard practice in the object-detection literature, overlap is measured as the intersection over union (IOU) of the proposed bounding box with a (human-labeled) ground-truth bounding box.

We implemented each object-localization model as a linear combination of features, where the features are obtained from running a pretrained convolutional network on the input region. We used the open-source VGG-f network pre-trained on Imagenet [7] to obtain 4096 features from the fc7 layer, and used ridge regression [8] to learn the coefficients and bias of the linear model. We trained the ridge regression model on features extracted from training-image crops that partially or completely overlap ground-truth boxes.

Object-Refinement Models: Situate similarly learns category-specific object-refinement models from the same training crops used to learn object-localization models. These refinement models-based on the "bounding-box regression" approach described in [9]-input an object proposal and output a new, "refined" object proposal that is predicted to have higher IOU with a ground-truth bounding box of the given category. Like the object-localization models described above, each category-specific object-refinement model is a linear combination of 4096 features from the pre-trained VGG fc7 layer. As we will describe below, on a new test image the refinement models will be applied to object proposals whose predicted overlap score is above a pre-set threshold. We found these refinement models to be crucial to Situate's ability to localize objects.

Relationship Model: For a given situation query, Situate learns a relationship model that represents the expected spatial relationships among the relevant objects in that situation. The relationship model is a multivariate Gaussian distribution learned from human-labeled bounding boxes in training images. The variables in the distribution are bounding-box parameters - center coordinates, area ratio (i.e, area of box divided by area of image), and aspect ratio-from the relevant objects in a given situation. For example, for the Walking the Dog situation shown in Figure 11 a), the variables are the bounding-box parameters of the Dog-Walker, Dog, and Leash boxes. As we will describe below, when Situate is run on a new image, each time it makes a candidate detection of one or more relevant objects, it conditions the relationship model on those detections to narrow the expected location, shape, and size of the related objects.

Priors on Object Size and Shape: Situate also learns a model capturing prior expectations of each relevant object category's size (area ratio) and shape (aspect ratio). These expectations are learned by fitting the area ratios and aspect ratios of ground-truth boxes as independent category-specific log-normal distributions. Log-normals are a better fit for the distribution of these values than normal distributions because the former are always positive and give more weight to smaller values. Note that our system does not learn prior distributions over bounding-box location, since we do not want the system to model photographers' biases to put relevant objects near the center of the image.

Fine-Tuned Faster-RCNN: Before starting its run on a test image, Situate runs a fine-tuned version of faster-RCNN on the test image to create a "prior" set of potential object proposals (to be described in the next section). Faster-RCNN [1] is a widely-used deep convolutional network that is trained 
to propose bounding boxes and score them with respect to given object categories; it has been shown to achieve stateof-the-art performance on object detection. We used an opensource version of Faster-RCNN [10] that was pre-trained on the Pascal VOC dataset, and we used Situate's training set to fine-tune it for the Dog-Walker, Dog, and Leash categories.

\section{B. Running Situate on a Test Image}

After the models described above have been learned from training data, Situate is ready to run on new ("test") images. The input to Situate is an image and the program's output is (1) a situation match score that measures Situate's assessment of this image as an instance of the given situation, and (2) a set of "groundings"- detections of situation components in the Workspace, as illustrated in Figure 1(c).

The detailed process by which Situate runs on a test image is illustrated in Figure 2, which shows visualizations of eight time-slices from a run of the program using the Walking the Dog situation of Figure 1(a).

Figure 2(a): The figure shows Situate's state before any agents have run. The Workspace contains the unprocessed image. The gray squares shown below the Workspace represent the probability distributions over location for each object category. The uniform gray indicates that these are initially uniform distributions. Once an object is detected, these distributions will be updated to be conditioned on that detection according to the learned relationship model. As we described above, the system also maintains probability distributions (not shown here) for aspect ratio ("shape") and area ratio ("size") of each object category. Initially these shape and size distributions are set to the learned independent priors for each category, but once an object is detected these distributions will also be conditioned on that detection.

As we described above, Situate will attempt to ground each relevant object (here, Dog-Walker, Dog, and Leash) in the Workspace via the action of agents. The system maintains an agent pool - a collection of agents waiting to run-and selects one of them at random to run at each time step.

In order to balance exploration for new object proposals with "exploitation" and refinement of known, promising object proposals, Situate has three types of agents: explorers, refiners, and RCNN-priors. An explorer agent chooses an object category from the list of relevant categories (here, Dog-Walker, Dog, and Leash), and samples from that category's current location, shape, and size distributions to create an object proposal. A refiner attempts to improve an already-existing object proposal. An RCNN-prior agent proposes one of the bounding boxes that were computed prior to Situate's run by running our fine-tuned faster-RCNN on the test image. We found that all three of these types of agents were needed in order for Situate to work well. The importance of explorer agents will be seen in Section VI when we compare Situate's performance with that of faster-RCNN alone.

The agent pool is initialized with $P$ RCNN-prior agents for each object category-these represent the $P$ highestconfidence boxes for each category, as computed and scored by faster-RCNN-and $P^{\prime}$ explorer agents. In the experiments described in Section $\mathrm{V}$, we set $P=10$ and $P^{\prime}=30$ - that is, 10 RCNN-priors per category, and 30 explorer agents (each of which will choose a category at random when it runs). These values were chosen via experiments on a validation subset of the training images.

Once an agent runs, it is permanently deleted from the pool. Every time an explorer agent is run and deleted, a new explorer agent is added to the pool, in addition to any follow-up refiner agents that were added by previously run agents.

The challenging aspects of this particular test image are (1) to identify the correct person as the dog-walker and (2) to locate the small, hard-to-see leash.

Figure 2(b): At time step 1 an explorer agent is selected from the pool to run. It chooses the Dog-Walker category, and samples from the location, size, and shape distributions of that category to produce a proposal, represented as a white box in the Workspace labeled "Dog-Walker?" (and a corresponding blue box in the location distribution). The explorer agent evaluates this proposal via two measures: internal and external support. The internal support is the Dog-Walker object-localization model's prediction of overlap between this proposal and a ground-truth dog-walker. The external support is a function that uses the learned relationship model to measure how well this proposal fits in with other detections that have been made (none so far in this example). These two measures are combined into a total support measure, which reflects the system's current judgement of the quality of this proposal for the given situation. If the internal support is greater than a pre-defined threshold, the proposal will be marked for refinement; if the total support is above a second threshold, the proposal will be accepted as a detection. Since internal support is very low for this particular Dog-Walker proposal, it will be discarded without followup. (Due to space constraints in this paper, the specific forms of the internal, external, and total support functions, along with values of the thresholds we used, will be given in an online supplementary information section when this paper is published.)

Figure 2(c): At time step 3 an RCNN-prior agent is selected to run. It creates a Dog-Walker proposal (in fact, this is fasterRCNN's most confident "Dog-Walker" box for this image). This proposal's internal support is high enough for the system to create a detection (red box in the Workspace; corresponding red box in the location distribution). A detection is a data structure in the Workspace indicating that the system is confident that it has located a particular object. The relationship model is now conditioned on this detection, yielding new location distributions for Dog and Leash, shown in the boxes below the Workspace. (The size and shape distributions for these categories, not shown here, are also conditioned on the detection.) Agents will now increasingly focus on searching for objects in higher-probability areas of these distributions (shown as white regions in the location distributions). Note that the Dog-Walker location distribution is still uniform, since no dogs or leashes have been detected yet. The only problem is that the program has identified the wrong person as the dog- 


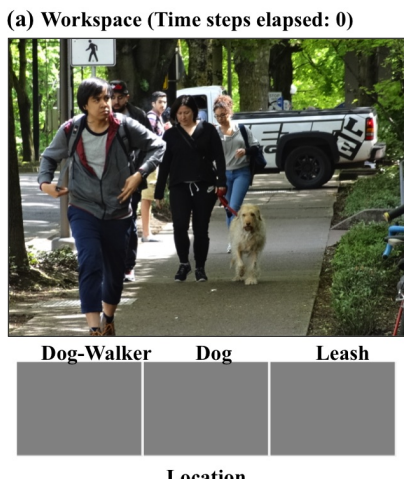

Location

(e) Workspace (Time steps elapsed: 14)
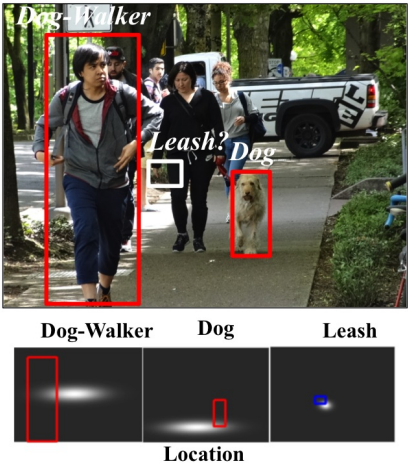

(b) Workspace (Time steps elapsed: 1)

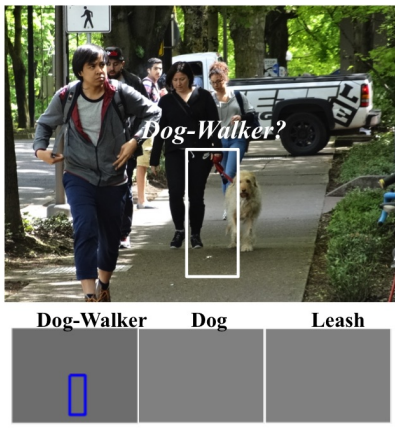

Location

(f) Workspace (Time steps elapsed: 16)
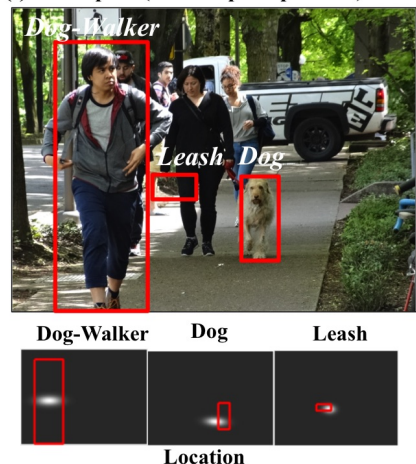

(c) Workspace (Time steps elapsed: 3 )
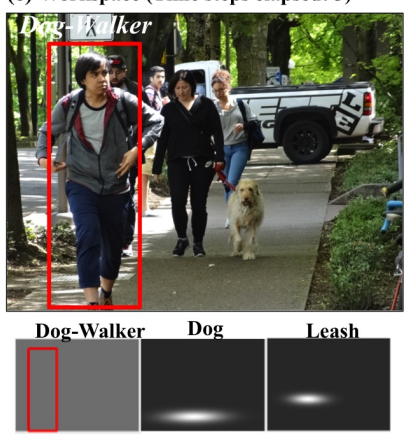

Location

(g) Workspace (Time steps elapsed: 31)
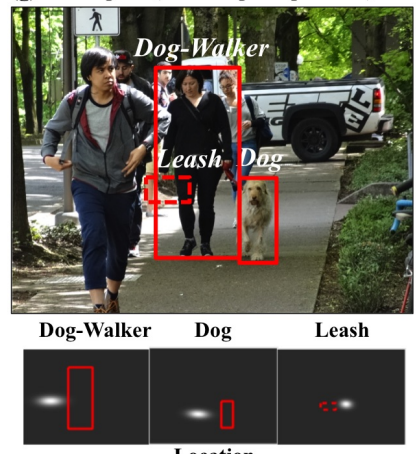

(d) Workspace (Time steps elapsed: 5)

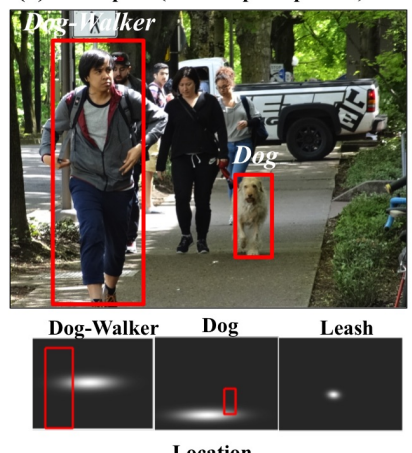

(h) Workspace (Time steps elapsed: 89)

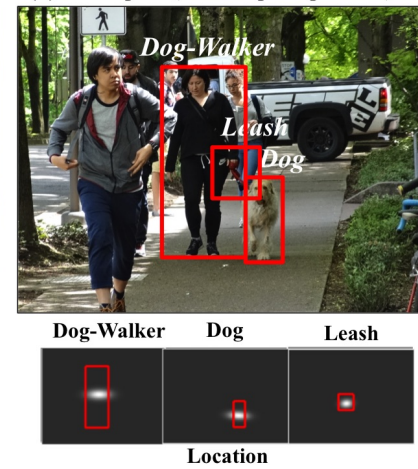

Fig. 2. (Best viewed in color.) Visualization of eight time steps in a run of Situate. See text for explanation.

walker. This will be corrected in subsequent time steps using additional information discovered by the program.

Figure 2(d): At time step 5 an RCNN-prior agent creates a Dog detection. The location distributions (as well as size and shape distributions) for each category are now conditioned on the detections of the other categories. In particular, notice that the Dog-Walker location distribution has been conditioned on the Dog detection, and that the current Dog-Walker detection is offset from the center of that distribution. Similarly, the new Dog detection is offset from the center of the Dog location distribution, which is conditioned on the Dog-Walker. In short, these detections do not support each other strongly. Even though both Dog-Walker and Dog have been detected, these (and likewise any detection) are treated as provisional until the end of the run: agents will continue to search for better-fitting alternatives.

Figure 2(e): At time step 14, an explorer agent samples from Leash distributions to produce a proposal (white box). While the internal strength of this proposal is not high enough for it to be a detection, a refiner agent is created to improve it, and is added to the agent pool.

Figure 2(f): The refiner agent runs at time step 16, creating a leash detection. (False-positive leash detections are very commonly made, both by faster-RCNN and by Situate.) The location distributions for each category are updated to reflect this new detection. While the Dog-Walker and Leash detections strongly support each other, the Dog detection does not fit in as well.
Figure 2(g): An explorer agent has proposed an alternative Dog-Walker, and due to its high internal support as well as external support from the Dog detection, this new DogWalker has higher total support than the previous Dog-Walker detection, and has replaced it. The relationship model has been updated to reflect the new set of detections. This causes the existing Leash detection to lose much of its external (and thus total) support; its weakness is illustrated by the dashed line around it.

Figure 2(h): A stronger Leash detection has replaced the weaker one, and all three detections now strongly support one another (each can be seen to be at the center of its location distribution). This mutual context has helped the system identify the correct dog-walker as well as to locate the small, hard-to-see leash. The run ends at this point, since all three objects have been detected. (If not all objects are detected before a pre-set maximum number of time steps, the run stops.)

At the end of a run, the system computes the situation match score as a function of the total support of each detection. In the current version of Situate, we define the situation match score as the geometric mean over the "padded" total support values of detections in the Workspace, where we "pad" the total support values by adding 0.01 to each in order to avoid multiplication by zero. If no detection for a given object is made by the end of the run, the situation match score is set to the minimum value of 0.01 . We chose this padded geometric mean function as a simple way of combining total 
support scores, but will investigate alternative scoring methods in future work.

In summary, the following sketches the initialization and main loop of Situate.

Input: A test image.

Initialization: Initialize location, area-ratio, and aspectratio distributions for each relevant object category. The initial location distributions are uniform; initial area-ratio and aspect-ratio distributions are learned from training data. Initialize agent pool with explorer and RCNN-prior agents.

Main Loop: Repeat until all relevant objects are detected or at most for Max-Iterations:

1) Choose agent from agent pool at random.

2) Run agent and delete it from the agent pool (and if agent is an explorer, replace it in the agent pool).

3) Update relationship model, conditioned on current detections in the Workspace.

Return: Situation match score $S$, where

$$
S=\left[\prod_{i=1}^{n}\left(\text { total-support }\left(d_{i}\right)+0.01\right)\right]^{\frac{1}{n}}
$$

where $n$ is the number of detections in the Workspace, and $d_{i}$ is the $i$ th detection.

For the experiments described in this paper, we used MaxIterations $=300$. That is, at most, 300 agents are run including explorers, refiners, and RCNN-priors.

In designing Situate's architecture, we were inspired by Hofstadter et al.'s idea of modeling perception as a "parallel terraced scan" [6], in which many possible exploration paths are pursued in parallel, but not all at the same speed or to the same depth. Moreover, the exploration is "active" in the sense that information is used as it is gained to continually modify the resources given to possible paths. An advantage of such an approach is balancing the need to explore many possibilities while still avoiding exhaustive evaluation of possible situation configurations. Like the codelets in the architecture of [6], our (serially run) architecture approximates such a parallel search strategy by interleaving many independent agents. In principle, many of these explorations could be performed in parallel. Furthermore, splitting up "explorers" and "refiners" allows the system to balance time spent on bottom-up exploration with time on focused follow-ups.

Due to space constraints, we omit some details of Situate (e.g., the detailed forms of the external and total support functions, thresholds for detections) and the other methods. We will provide these details in an online supplementary materials section, along with our code and all training and test data, upon publication of this paper.

We hypothesize that the approach we have described above will have superior performance on grounding elements of situations, and thus on ranking images, than methods that do not use this kind of active approach, assuming the same amount of training data. We also hypothesize that our method will be able to achieve this performance with significantly fewer object-proposal evaluations than non-active methods.

\section{RELATED WORK}

Here we describe some of the recent approaches most closely related to Situate's goals and architecture. Closely related to our work is the approach of Johnson et al. [3] for semantic image retrieval via "scene graphs." We describe this method in Section $\mathrm{V}$ and compare its performance to that of Situate in Section VI

Another widely studied image-understanding task is that of "grounding referential expressions" (e.g., [11]-[13]). Given a phrase such as "the brown dog next to the woman wearing sunglasses," the task is to locate the object being referred to, by grounding each object and relation in the phrase. Like the scene-graph task described above, research on this task has focused on specific "free-form" phrases rather than more abstract situation descriptions. The open-ended nature of the task makes it very difficult, and accuracies reported on large datasets have remained low to date. A related task is that of detecting visual relationships in images (e.g., [14]); to our knowledge, the literature on this task has focused almost exclusively on pairwise relationships (e.g. "dog riding surfboard"), rather than multi-object visual situations.

Our situation-retrieval task shares motivation but contrasts with the well-known tasks of "event recognition" or "action recognition" in still images (e.g., [15]-[17]). These latter tasks consist of classifying images into one of several event or action categories, without the requirement of localizing objects or relationships. A related task, dubbed "Situation Recognition" in [18], requires a system to, given an image, predict the most salient verb, along with its subject and object ("semantic roles" [19]).

Our task also contrasts with recent work on automatic caption generation for images (e.g., [20]), in which image content is statistically associated with a language generator. The goal of caption-generation systems is to generate a description of any input image. Even the versions with "attention" (e.g., [21]), which are able to highlight diffuse areas corresponding roughly to relevant objects, are not generally able to recognize and locate all important objects, relationships, and actions, or more generally to recognize abstract situations.

While the literature cited above does not include active detection methods such as Situate that involve feedback, there has been also considerable work on active object detection (e.g., [22], [23]), often in the context of active perception in robots [24] and modeling visual attention [25], [26]. More recently, several groups have framed active object detection as a Markov decision process and use reinforcement learning to learn a search policy (e.g., [27]).

This section has given a sampling of recent work most closely related to Situate. While our work shares motivation with some of these efforts, the specific problem we are addressing (visual situation retrieval) and method (active grounding of situation components) is, to our knowledge, unique in the literature. 


\section{DATASETS}

The computer vision community has created several important benchmark datasets for object recognition and detection (e.g., [2], [28]) and for some of the other tasks described in the previous section that combine vision and language (e.g., [29], [30]). None of these offers the kind of data that we needed for our situation-retrieval task-that is, collections of numerous instances of specific multi-object situations, in which the objects are localized with ground-truth bounding boxes. (For example, the ImSitu "Situation Recognition" dataset is organized around verbs such as carrying, jumping, and attacking, each with one subject (e.g., "dog jumping") and some with one additional object that the subject acts upon (e.g., "man carrying baby").

For our preliminary work with Situate, we developed a new dataset representing the "Walking the Dog" situation. We chose this situation category because it is reasonably easy to find sufficient varied instances to train and test our system, and these instances offer a variety of interesting challenges. This dataset, the "Portland State Dog-Walking Images," contains 500 positive instances of the Dog-Walking situation. These positive instances are photographs taken by members of our group, and in each we labeled (with bounding boxes) the Dog, Dog-Walker, and Leash. Each image contains only one of each target object, but many also contain additional (non-dogwalking) people, along with cars, buildings, trees, and other "clutter." The challenges of this dataset include determining which person is the dog-walker, as well as locating dogs (often small, and sometimes partially occluded) and leashes (which are very often difficult, based on visual features alone, to distinguish from other line-like structures in an image), and deciding if the configuration of these three objects fits the learned dog-walking situation. For the experiments described below, we split the 500 images into a 400-image training set and a 100-image test. We also created a negative set of 400 images selected from the Visual Genome dataset [29], including images in which people interact with dogs in nondog-walking situations, along with images with people but no dogs, dogs but no people, and neither.

\section{Methods}

We performed experiments to evaluate Situate's image retrieval and situation grounding abilities. We assess the importance of Situate's learned relationship models by comparing with two baseline methods as well as with the Image Retrieval using Scene Graphs (IRSG) method of Johnson et al. [3]. In each method, we performed any necessary training using the same training set that we used for Situate. All methods are tested on the "Portland State Dog Walking" test set.

Baseline Methods: The first baseline, which we call the Uniform method, is identical to Situate except that explorer agents always sample locations and bounding box parameters uniformly rather than using a learned relationship model. When creating an object proposal, an explorer agent chooses a center location by sampling uniformly across the entire image, and chooses area and aspect ratios by sampling uniformly over fixed ranges. The second baseline uses our fine-tuned version of Faster-RCNN (described in Section II-A above). We ran the fine-tuned Faster-RCNN network on each test image (positive and negative), and selected the highest scoring bounding box (as scored by Faster-RCNN) corresponding to each of the three relevant object categories. Analogous to Situate, we defined the Situation Match Score as the padded geometric mean of the scores assigned to these bounding boxes by Faster-RCNN. Our goal was to see how well this specially trained FasterRCNN model could be used to perform situation retrieval by simply relying on the top-scoring boxes for each relevant object, absent of any relationship model or active search.

IRSG Method: We also compare Situate's performance that of Johnson et al.'s "Image Retrieval using Scene Graphs" (IRSG) method [3]. A scene graph is a graphical representation of objects, attributes, and relationships that encode an image or image region, or desired image content-e.g., "a tall man wearing a white baseball cap." IRSG is similar to Situate in that it scores an input image as to how well it instantiates a query description (represented as a scene graph), and uses these scores to rank images in a collection. Moreover, IRSG computes its score by attempting to ground components of the query scene graph in the input image. IRSG first runs the geodesic object proposal method of [31] to create a set of non-category-specific object proposal bounding boxes (on the order of several hundred per image). IRSG then uses R-CNN [9] to give each bounding box multiple "appearance" scoresone for each object category in the scene graph. Then, for each possible pair of bounding boxes, IRSG uses a set of Gaussian mixture models (GMM), learned from training examples, to score the pair on each relationship in the scene graph. The unary object appearance scores and binary relationship scores are used in a conditional random field model defined over a factor graph representing the query. As a simple example, for the query "woman next to man wearing hat," the system would give an appearance score to each bounding box for "woman," "man," and "hat," and then give a relationship score to each pair of bounding boxes for each of the two relationships ("man wearing hat" and "woman next to man"), and see which configuration of object boxes and relationships minimizes the energy function defined by the conditional random field.

We obtained the source code for IRSG from the authors of [3] and adapted it in order to compare it with Situate and our other methods. Instead of geodesic object proposals scored by R-CNN, we used the top-scoring 300 boxes per category (DogWalker, Dog, Leash) from our fine-tuned version of FasterRCNN. Since IRSG is (as currently implemented) limited to pairwise relationships, we trained GMMs to represent three spatial relationships: Dog-Walker and Leash; Leash and Dog; and Dog-Walker and Dog. The conditional random field formulation and energy minimization was performed using the same algorithms that were described in [3]. In this way, each positive and negative test image in our set was scored with its final energy value, and images were ranked in order of increasing energy-i.e., the lowest energy image was ranked the highest. (In the other methods, images were ranked in order 
TABLE I

RESUlts For Single-IMAge RECALL@ $N$. Best RESUlts FOR EACH $N$ ARE IN BOLdFACE.

\begin{tabular}{|l|l|l|l|l|l|l|}
\hline $\mathrm{N}$ & 1 & 2 & 5 & 10 & 20 & 100 \\
\hline Situate & $\mathbf{0 . 3 7}(0.09)$ & $\mathbf{0 . 5 0}(0.07)$ & $\mathbf{0 . 5 6}(0.07)$ & $\mathbf{0 . 6 5}(0.04)$ & $\mathbf{0 . 7 7}(0.05)$ & $\mathbf{0 . 9 3}(0.02)$ \\
\hline Uniform & $0.29(0.07))$ & $0.32(0.07)$ & $0.44(0.06)$ & $0.54(0.04)$ & $0.65(0.03)$ & $0.79(0.03)$ \\
\hline Faster-RCNN & 0.24 & 0.25 & 0.28 & 0.38 & 0.54 & 0.91 \\
\hline IRSG & 0.24 & 0.24 & 0.27 & 0.37 & 0.55 & 0.87 \\
\hline
\end{tabular}

of decreasing score- the highest scoring image was ranked the highest.)

Evaluation Metric: We ran each method on the 100 positive test images and the the 400 negative (non-dog-walking) test images chosen from the Visual Genome dataset. Our evaluation metric is Single-Image Recall @ $N$ (abbreviated $R @ N)$ : the probability that, if a single positive example were added to the set of negative examples, and the collection was ranked by situation match score (or energy, for IRSG), the positive example would be in the $N$ top-ranked images. For example, given our 100 positive and 400 negative test images, $R @ 10=0.65$ means that 65 out of 100 of the positive images would be in the top 10 ranked images if they were ranked alone with the 400 negative images.

\section{Results}

Table I gives the $R @ N$ values resulting from running the four different methods on the positive and negative test sets. While Faster-RCNN and our adapted version of IRSG are deterministic, the Situate and Uniform methods are stochastic. We performed 10 independent runs of each of these latter methods; each run consisted of running on all the positive and negative test images, and then computing $R @ N$. The values given in Table I for Situate and Uniform are the averages over these $R @ N$ values (with standard deviations in parentheses). It can be seen that Situate produced the highest $R @ N$ of any method for each value of $N$; for low values of $N$, which are typically the most important for retrieval tasks, Situate's $R @ N$ was substantially higher than any of the other methods. These results support our hypothesis that Situate's active grounding method, together with its learned models, will result in superior image retrieval performance than methods lacking these components.

\section{DISCUSSION}

We investigated the reasons for for Situate's superior performance by viewing the object detections produced by each method by the end of a run on each test image. As was illustrated by the run in Figure 2, Situate was considerably better at identifying the correct person as Dog-Walker and at locating hard-to-see leashes than the other methods. Moreover, Situate was better at locating small or partially occluded objects than the other methods. Also, in the case of fasterRCNN, even when it did locate a hard-to-see object, fasterRCNN often gave it low confidence, making the situationmatch score low. In contrast, due to its external support measure, Situate was able to assign higher confidence to such objects and thus score positive situation instances more highly than faster-RCNN.

All methods were susceptible to false-positive object detections with high confidence, but again, because of its incorporation of external support for object detections, Situate was less susceptible than RCNN and IRSG to giving high situation match scores to negative images. In principle IRSG should have also given higher energy (lower score) to such images due to its relationship models, but it was not very effective at doing so. We will investigate the reasons for its failures in detail in the near future.

While we have reported results on only one particular situation query ("Dog-Walking"), we believe that the mechanisms that lead to Situate's superior retrieval performance are general and scalable. Demonstrating this scalability and generality is the most important topic of our near-term future work.

\section{CONCLUSIONS AND FUtURE WORK}

In this paper we have described a preliminary study of Situate, a novel approach to semantic image retrieval. The results of this study have shown the promise of Situate's active situation-grounding architecture: our system's image-retrieval performance on the "Walking the Dog" situation strongly surpassed that of two baselines as well as a related imageretrieval system from the recent literature. We showed how Situate is able to use information as it is gained in order to focus its search, and to use the support of context in order to locate hard-to-detect objects (e.g., barely visible leashes, small dogs, partially occluded objects). In analyzing these results, we were able to understand some of the reasons for Situate's superior performance, as well as to identify some of its problems. This analysis underscores the important role of grounding situation elements as part of scoring an image.

Visual situation recognition and retrieval is a broad and difficult open problem in computer vision research, and the results we have presented highlight many avenues of future research. In the near term we plan to improve our algorithm in several ways: expanding the kind of object attributes that can be detected by agents (e.g., orientation and other pose features); expanding the types of relationships that can be identified (e.g., recognizing that two objects have the same orientation). In the work described here we used a multivariate Gaussian to capture spatial relationships among objects; this simple probabilistic model is very fast to learn and to sample from. We plan to experiment with more sophisticated relationship models while keeping in mind the tradeoff between sophistication and speed of computation. Most importantly, 
we will explore the ability of our algorithms to scale to larger datasets and to generalize to other situation categories.

In the longer term, we will focus on, among other extensions, being able to speed up our active search method via parallelization. Finally, one of our original motivations for this project was to create a system that can recognize visual analogies. For example, most people would consider images of a person running, riding a bicycle, sitting in a wheelchair, etc. while "walking" a dog to still be instances of the abstract Dog-Walking situation. This kind of recognition requires what Hofstadter and colleagues have called "conceptual slippage," in which the roles defining a situation can be fluidly filled by concepts semantically related to the prototype. Making appropriate conceptual slippages is at the heart of analogymaking, which itself is a core aspect of cognition [6].

The abilities of computer vision remain far from humanlevel visual understanding, but we believe that progress on the problem of situation recognition, particularly incorporating analogy-making, will play a pivotal role in giving computers the ability to make sense of what they see.

\section{ACKNOWLEDGMENTS}

We are grateful to Justin Johnson of Stanford University for sharing the source code for the IRSG project, and to NVIDIA corporation for donation of a GPU used for this work. Many thanks to Garrett Kenyon, Bryan Lee, Sheng Lundquist, Anthony Rhodes, Evan Roche, Rory Soiffer, and Robin Tan for discussions and assistance concerning this project. This material is based upon work supported by the National Science Foundation under Grant Number IIS-1423651. Any opinions, findings, and conclusions or recommendations expressed in this material are those of the authors and do not necessarily reflect the views of the National Science Foundation.

\section{REFERENCES}

[1] J. Ren, S., He, K., Girshic, R., Sun, "Faster R-CNN: Towards realtime object detection with region proposals," in Advances in Neural Information Processing Systems 28 (NIPS 2015), 2015.

[2] J. Deng, W. Dong, R. Socher, L.-J. Li, K. Li, and L. Fei-Fei, "ImageNet: A large-scale hierarchical image database," in 2009 IEEE Conference on Computer Vision and Pattern Recognition (CVPR), 2009, pp. 248-255.

[3] J. Johnson, R. Krishna, M. Stark, L.-J. Li, D. Shamma, M. Bernstein, and L. Fei-Fei, "Image retrieval using scene graphs," in IEEE Conference on Computer Vision and Pattern Recognition (CVPR), 2015, pp. 3668 3678.

[4] M. Neider and G. Zelinsky, "Scene context guides eye movements during visual search," Vision Research, vol. 46, no. 5, pp. 614-621, 2006.

[5] C. Summerfield and T. Egner, "Expectation (and attention) in visual cognition." Trends in Cognitive Sciences, vol. 13, no. 9, pp. 403-9, 2009.

[6] D. R. Hofstadter and M. Mitchell, "The Copycat project: A model of mental fluidity and analogy-making," in Advances in Connectionist and Neural Computation Theory, K. Holyoak and J. Barnden, Eds. Ablex Publishing Corporation, 1994, vol. 2, pp. 31-112.

[7] "Imagenet-VGG-f." [Online]. Available: http://www.vlfeat.org/ matconvnet/pretrained/

[8] "Matlab Ridge Regression." [Online]. Available: https://www. mathworks.com/help/stats/ridge.html

[9] R. Girshick, J. Donahue, T. Darrell, and J. Malik, "Rich feature hierarchies for accurate object detection and semantic segmentation," in IEEE Conference on Computer Vision and Pattern Recognition (CVPR), 2014, pp. 580-587.
[10] R. B. Girshick, "FasterRCNN Source Code," 2015. [Online]. Available: https://github.com/rbgirshick/py-faster-rcnn

[11] J. Mao, J. Huang, A. Toshev, O. Camburu, A. Yuille, and K. Murphy, "Generation and Comprehension of Unambiguous Object Descriptions," IEEE Conference on Computer Vision and Pattern Recognition (CVPR), pp. 11-20, 2016.

[12] V. K. Nagaraja, V. I. Morariu, and L. S. Davis, "Modeling context between objects for referring expression understanding," in Proceedings of the European Conference on Computer Vision, vol. 9908 LNCS, 2016, pp. 792-807.

[13] A. Rohrbach, M. Rohrbach, R. Hu, T. Darrell, and B. Schiele, "Grounding of Textual Phrases in Images by Reconstruction," in Proceedings of the European Conference on Computer Vision, 2016, pp. 817-834.

[14] C. Lu, R. Krishna, M. Bernstein, and L. Fei-Fei, "Visual relationship detection with language priors," in Proceedings of the European Conference on Computer Vision, 2016, pp. 852-869.

[15] G. Guo and A. Lai, "A survey on still image based human action recognition," Pattern Recognition, vol. 47, no. 10, pp. 3343-3361, 2014.

[16] L.-J. Li and L. Li Fei-Fei, "What, where and who? Classifying events by scene and object recognition," in International Conference on Computer Vision (ICCV). IEEE, 2007, pp. 1-8.

[17] L. Wang, Z. Zhe Wang, W. Wenbin Du, and Y. Qiao, "Object-scene convolutional neural networks for event recognition in images," in Conference on Computer Vision and Pattern Recognition (CVPR) Workshops. IEEE, 2015, pp. 30-35.

[18] A. Yatskar, M, Zettlemoyer, L., Farhadi, "Situation recognition: Visual semantic role labeling for image understanding," in Conference on Computer Vision and Pattern Recognition (CVPR). IEEE, 2016.

[19] S. Gupta and J. Malik, "Visual Semantic Role Labeling," arXiv:1505.04474, 2015

[20] O. Vinyals, A. Toshev, and S. Bengio, "Show and Tell: Lessons learned from the 2015 MSCOCO Image Captioning Challenge," IEEE Transactions on, 2015.

[21] K. Xu, J. Ba, R. Kiros, K. Cho, and A. Courville, "Show, attend and tell: Neural image caption generation with visual attention," in International Conference on Machine Learning, 2015, pp. 2048-2057.

[22] B. Alexe, N. Heess, Y. Teh, and V. Ferrari, "Searching for objects driven by context," in Advances in Neural Information Processing Systems, 2012, pp. 1-9.

[23] A. Gonzalez-Garcia, A. Vezhnevets, and V. Ferrari, "An active search strategy for efficient object class detection," in Conference on Computer Vision and Pattern Recognition (CVPR). IEEE, 2015, pp. 3022-3031.

[24] D. H. Ballard, "Animate vision," Artificial Intelligence, vol. 48, no. 1, pp. 57-86, 1991.

[25] J. Ba, V. Mnih, and K. Kavukcuoglu, "Multiple object recognition with visual attention," arXiv:1412.7755, 2014.

[26] V. Mnih, N. Heess, A. Graves, and K. Kavukcuoglu, "Recurrent models of visual attention," in Advances in Neural Information Processing Systems (NIPS), 2014, pp. 2204-2212.

[27] J. C. Caicedo and S. Lazebnik, "Active object localization with deep reinforcement learning," in International Conference on Computer Vision (ICCV). IEEE, 2015, pp. 2488-2496.

[28] M. Everingham, L. Gool, C. K. I. Williams, J. Winn, and A. Zisserman, "The Pascal visual object classes (VOC) challenge," International Journal of Computer Vision, vol. 88, no. 2, pp. 303-338, 2010.

[29] R. Krishna, Y. Zhu, O. Groth, J. Johnson, K. Hata, J. Kravitz, S. Chen, Y. Kalantidis, L.-J. Li, D. A. Shamma, and Others, "Visual genome: Connecting language and vision using crowdsourced dense image annotations," International Journal of Computer Vision, vol. 123, no. 1, pp. 32-73, 2017.

[30] T. Y. Lin, M. Maire, S. Belongie, J. Hays, P. Perona, D. Ramanan, P. Dollár, and C. L. Zitnick, "Microsoft COCO: Common objects in context," in European Conference on Computer Vision (ECCV), may 2014, pp. 740-755.

[31] P. Krähenbühl and V. Koltun, "Geodesic object proposals," in Proceedings of the European Conference on Computer Vision (ECCV 2014). Springer, Cham, 2014, pp. 725-739. 